\section{ASPIRIN PLATELET REACTIVITY AS A DETERMINANT OF LONG-TERM SURVIVAL IN NON ST-ELEVATION MYOCARDIAL INFARCTION (NSTEMI) PATIENTS}

${ }^{1}$ Nazish Khan*, ${ }^{2}$ Andrew Smallwood, ${ }^{2}$ Ben Wrigley, ${ }^{2}$ Saib Khogali, ${ }^{2}$ Shahzad Munir, ${ }^{3}$ James Cotton. ${ }^{1}$ The Royal Wolverhampton Hospitals NHS Trust; ${ }^{2}$ Department of Cardiology, The Royal Wolverhampton Hospitals NHS Trust; ${ }^{3}$ Department of Cardiology, Heart and Lung Centre, New Cross Hospital, Wolverhampton, UK

\subsection{6/heartjnl-2019-BCS.146}

Background/Introduction Aspirin, the most commonly prescribed anti-platelet agent, forms a cornerstone of management in patients with established cardiovascular disease. Despite proven efficacy, variability of aspirin response has long been recognised, with early studies suggesting rates of high on treatment platelet reactivity (HPR) as high as $5-45 \%$. True "aspirin resistance", when defined by strict platelet function testing is present in approximately $2-5 \%$ and is associated with poorer outcomes at 1-2 years in ACS patients. We sought to investigate whether the impact of VerifyNow determined response to aspirin, even at levels previously thought to indicate adequate aspirin response, was prognostically important in terms of all-cause mortality at 10 years.

Methods A prospective single centre analysis of 224 consecutive troponin positive NSTEMI patients undergoing coronary angiography. All aspirin naive patients were loaded with 300 $\mathrm{mg}$ aspirin and maintained on $75 \mathrm{mg}$ daily. Those admitted on aspirin continued on $75 \mathrm{mg}$ daily. Citrated blood samples were obtained at the time of coronary angiography and the VerifyNow Aspirin assay was utilised to determine aspirin reaction units (ARU).

The primary end point of our study was an assessment of survival at 10 years, expressed as all-cause mortality. Long term survival data were obtained using Office of National Statistics data.

Results Baseline characteristics and cardiovascular risk factors are described in table 1 . The mean time between the administration of an aspirin loading dose (or admission on aspirin) to angiography was $4.9 \pm 2.7$ days.

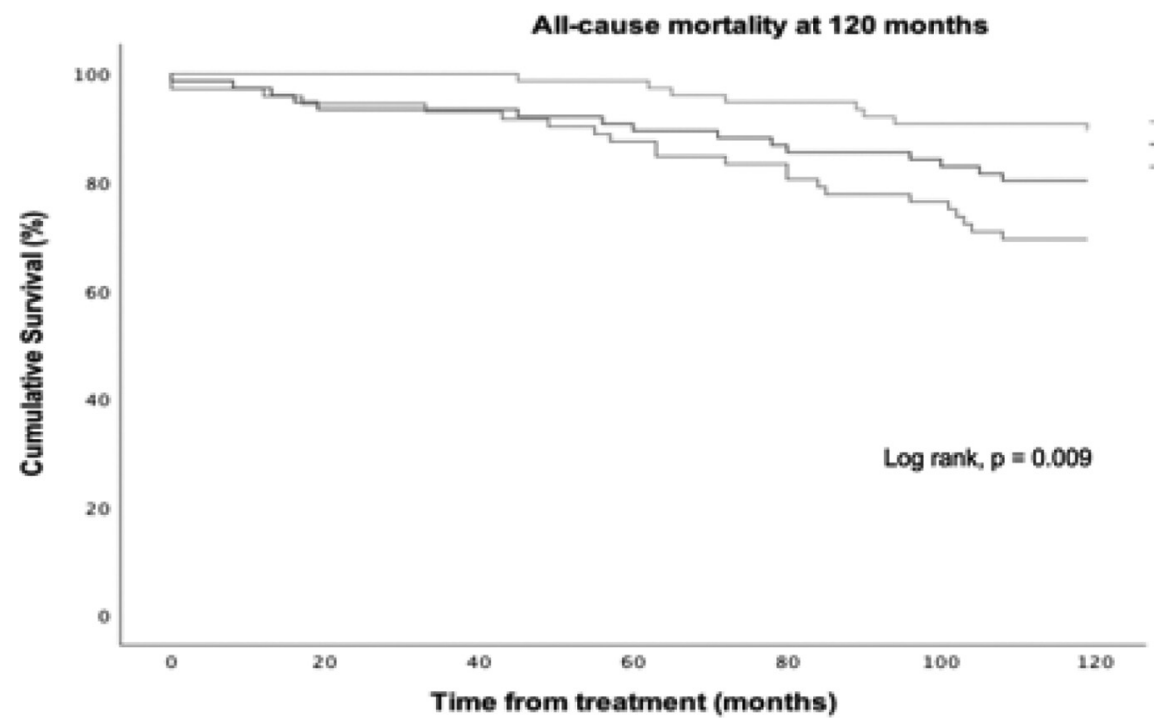

Abstract 149 Figure 1 Degree of HPR as a predictor of long-term survival outcomes assessed using VerifyNow Aspirin assay and expressed as aspirin reaction units (ARU)

Abstract 149 Table 1 Baseline Characteristics of troponin positive ACS patients expressed according to tertiles.

\begin{tabular}{|l|l|l|l|l|}
\hline & $\mathrm{T} 1(\mathrm{n}=76)$ & $\mathrm{T} 2(\mathrm{n}=76)$ & $\mathrm{T} 3(\mathrm{n}=72)$ & $\mathrm{p}$-value \\
\hline Age & $61.12 \pm$ & $64.46 \pm$ & $64.90 \pm$ & 0.06 \\
& 11.00 & 10.47 & 10.40 & \\
\hline Sex (male) & $52(68)$ & $48(63)$ & $52(72)$ & 0.494 \\
\hline Risk Factors & & & & \\
\hline Diabetes & $9(12)$ & $16(21)$ & $20(28)$ & 0.057 \\
\hline Hypertension & $36(47)$ & $46(61)$ & $43(60)$ & 0.153 \\
\hline Ex-Smoker & $23(30)$ & $28(37)$ & $27(38)$ & 0.603 \\
\hline Previous MI & $15(20)$ & $22(29)$ & $21(29)$ & 0.346 \\
\hline Previous CABG & $3(4)$ & $5(7)$ & $4(6)$ & 0.779 \\
\hline Previous TIA/Stroke & $3(40$ & $7(9)$ & $5(7)$ & 0.440 \\
\hline Positive FHx & $32(42)$ & $37(49)$ & $32(44)$ & 0.717 \\
\hline Hypercholesterolaemia & $33(43)$ & $45(59)$ & $37(51)$ & 0.125 \\
\hline
\end{tabular}

Values are expressed as mean \pm standard deviation, frequency $(\%)$. MI = myocardial infarction, CABG = coronary artery bypass graft, $\mathrm{TIA}=$ transient ischaemic attack, FHx $=$ family history 
Platelet aggregation results, expressed as VN ARU were divided into tertiles:

- $\mathrm{T} 1$ (ARU 363 - 405) (n=76),

- T2 (ARU 406 - 436) ( $=76)$,

- T3 (ARU 437 - 596) ( $\mathrm{n}=72)$.

Figure 1, demonstrates that all-cause mortality is significantly different between groups, (log-rank, $\mathrm{P}=0.009)$, with higher ARU values being associated with increased mortality.

A co-regression analysis indicates that, ex-smoker status $(\mathrm{HR}=3.60,95 \% \mathrm{CI}[1.47-8.85] \mathrm{p}=0.005)$, in addition to $\mathrm{HPR}$ on presentation $(\mathrm{HR}=3.13,95 \% \mathrm{CI}[1.38-7.10] \mathrm{p}$ $=0.006)$, were statistically significant predictors of mortality at 10 years.

Conclusion Our study demonstrates that ARU values less than the previously defined cut off 550 are associated with reduced survival at 10 years.

There are currently a number of pharmacological strategies aimed at reducing the considerable ongoing ischaemic risk in patients admitted with NSTEMI, including prolonged dual antiplatelet therapy, prolonged monotherapy with a P2Y12 inhibitor or low dose rivaroxaban. In order to improve longterm prognosis, NSTEMI patients with suboptimal, but not strict HPR to aspirin, might benefit from alternative and/or adjunctive antithrombotic treatment options. We suggest that this hypothesis warrants further study.

Conflict of Interest None

\section{COMPARING THE LENGTH OF STAY, EFFICACY AND SAFETY OF A NEW 1 HOUR "RULE-OUT" PATHWAY TO STANDARD CARE FOR PATIENTS PRESENTING TO THE EMERGENCY DEPARTMENTS WITH A SUSPECTED ACUTE CORONARY SYNDROME AT BRIGHTON AND SUSSEX UNIVERSITY HOSPITALS NHS TRUST}

${ }^{1}$ Nicolas Buttinger*, ${ }^{2}$ Daniel White, ${ }^{1}$ Jonathan Shurlock, 'Lucy Blows, ${ }^{1}$ Maureen Dooley. ${ }^{1}$ Brighton and Sussex University Hospitals NHS Trust; ${ }^{2}$ Royal Surrey County Hospital Guildford

10.1136/heartjnl-2019-BCS.147



Abstract 150 Figure 1 Time from arrival to discharge home from the ED in all patients presenting with suspected ACS $(p=0.012)$

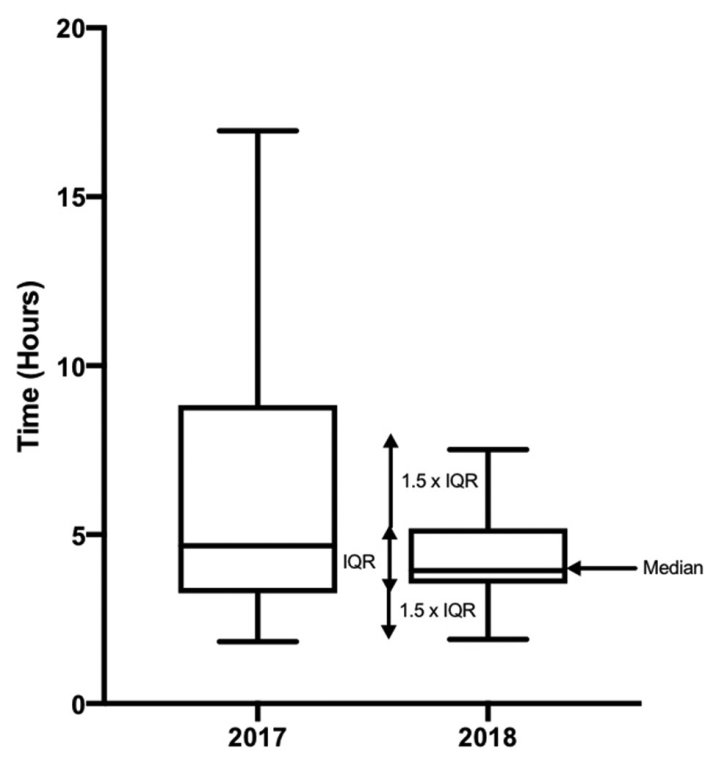

Abstract 150 Figure 2 Time from arrival to discharge home from the ED for patients with a To $>12 \mathrm{ng} / \mathrm{L}(\mathrm{p}=0.0359)$

Aim To compare the length of stay, efficacy and safety of a new $0 \mathrm{~h} / 1 \mathrm{~h}$ algorithm, utilising a high sensitivity troponin $\mathrm{T}$ (HsTnT) assay, with the previous standard 3 hour troponin at the Brighton and Sussex University Hospitals NHS Trust (BSUH) Emergency Departments (ED).

Methods The 2015 ESC guidelines approved a 1 hour algorithm used in conjunction with a HsTnT assay and clinical assessment to safely "rule-out" an NSTEMI in patients presenting to the ED with suspected ACS. We collected data on all patients attending the ED with suspected ACS in April 2017-pre and April 2018-post pathway implementation. The pathway triages patients into "rule-in", "rule-out" and "observation" groups. The initial pathway we developed included a lower cut-off than the ESC algorithm, $<3$ versus (v) $<5 \mathrm{ng} / \mathrm{L}$ for the initial troponin value (To) in order to allocate a patient to the "rule-out" group. 30-day all-cause mortality and major adverse cardiovascular events (MACE) were collected for both years. The final diagnosis was collected from discharge summaries for those admitted from the ED.

Results In April 2017 v April 2018 there were 391 v 491 patients who presented to the ED with suspected ACS. Mean age of 61 v 57 years and 59.3\% v 58.5\% male.

There was a reduction with the new pathway in the median time from ED arrival to discharge for all discharged patients 3.92 hours [IQR $=3.18-6.23]$ v 3.78 hours [IQR = $3.2-5.17]$. In patients triaged to the "rule-out" group, $281 \mathrm{v}$ 392 , the median time from ED arrival to discharge was 3.82 hours [IQR $=3-5.87]$ v 3.63 hours [IQR $=3.07-3.98$ ] (see figure 1). In those who had a To value $>12 \mathrm{ng} / \mathrm{L}, 63 \mathrm{v} 71$ patients, and subsequently discharged home, there was a significant reduction in the median time from ED arrival to discharge from 4.67 hours $[\mathrm{IQR}=3.27-8.83]$ to 3.93 hours [IQR = 3.55-5.18] (see figure 2).

In April 2018 65.6\%, 24.4\% and 10\% of patients with a suspected ACS were triaged to the "rule-out", "observation" and "rule-in" groups respectively. A diagnosis of ACS was made in $10.83 \%$ and $63.3 \%$ of patients in the "observation" and "rule-in" groups respectively.There was a significant reduction in the proportion of patients admitted $27.9 \% \quad \mathrm{v} \quad 20 \%$ with an increase in those admitted being given a primary 